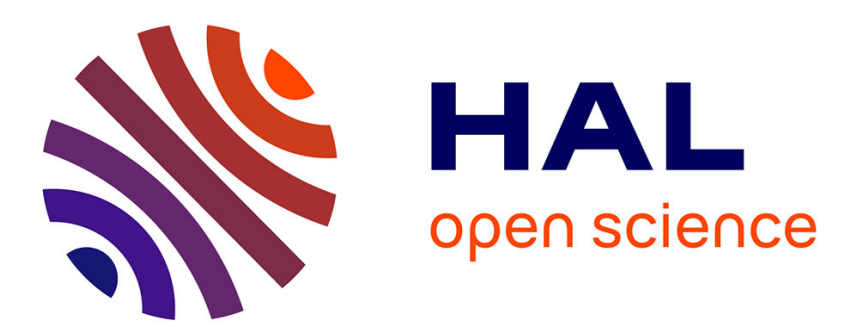

\title{
Le travail éducatif parental dans les classes moyennes et supérieures : deux modes contrastés d'encadrement des pratiques et des choix des enfants
}

Agnès van Zanten

\section{- To cite this version:}

Agnès van Zanten. Le travail éducatif parental dans les classes moyennes et supérieures: deux modes contrastés d'encadrement des pratiques et des choix des enfants. Informations sociales, 2009, 4 (154), pp.80 - 87. 10.3917/inso.154.0080 . hal-01521701v2

HAL Id: hal-01521701

https: / hal-sciencespo.archives-ouvertes.fr/hal-01521701v2

Submitted on 7 Nov 2017

HAL is a multi-disciplinary open access archive for the deposit and dissemination of scientific research documents, whether they are published or not. The documents may come from teaching and research institutions in France or abroad, or from public or private research centers.
L'archive ouverte pluridisciplinaire HAL, est destinée au dépôt et à la diffusion de documents scientifiques de niveau recherche, publiés ou non, émanant des établissements d'enseignement et de recherche français ou étrangers, des laboratoires publics ou privés. 


\title{
Le travail éducatif parental dans les classes moyennes et supérieures : deux modes contrastés d'encadrement des pratiques et des choix des enfants
}

\author{
Agnès van Zanten
}

De quelle manière les parents encadrent-ils les pratiques extrascolaires et les choix d'établissement et d'orientation de leurs enfants? Dans les classes moyennes et supérieures, cet encadrement semble épouser deux modalités : un encerclement protecteur autour de l'enfant visant à recréer un " entre soi " de classe ; l'accompagnement par le dialogue qui peut montrer les limites de la socialisation délibérative.

Si toutes les catégories sociales sont concernées par le travail d'éducation auprès des enfants, celui-ci revêt cependant des formes plus élaborées dans les classes moyennes et supérieures que dans les classes populaires, en raison de l'inégale répartition des ressources, mais aussi de conceptions différentes de ce que constitue un bon encadrement éducatif. Les parents de milieu populaire adhèrent en effet majoritairement à l'idéal-type du " développement naturel ", qui favorise une faible intervention parentale, alors que ceux des classes moyennes et supérieures se retrouvent davantage dans l'idéal-type d'une " inculcation systématique " (Lareau, 2003).

Un examen plus détaillé permet néanmoins de constater, dans la continuité des travaux de Basil Bernstein $(1975,2007)$, que ce dernier modèle com-porte des variantes. En nous appuyant sur les résultats d'une enquête par entretiens sur les choix scolaires, menée auprès de 165 parents urbains appartenant, en très grande majorité, aux catégories "cadres et professions intellectuelles supérieures " et " professions intermédiaires » de la nomencla-ture Insee (van Zanten, 2009), nous distinguerons ici deux modalités idéal-typiques d'encadrement des pratiques et des choix des enfants : l'encerclement et l'argumentation. 


\section{L'encerclement ou la réduction de l'univers du souhaitable}

L'encerclement apparaît comme la modalité dominante d'encadrement des enfants parmi les couples dont au moins un des conjoints, le plus souvent le père, est cadre du privé ou membre de certaines professions intellectuelles supérieures. On peut qualifier ces familles de " néopositionnelles ", parce qu'elles se situent à la fois du côté du respect de l'ordre et de la hiérarchie, comme les indépendants traditionnels, et du côté du marché et du management flexible, comme les nouveaux salariés du secteur privé (Bernstein, 2007 ; Gombert, 2008). Ces parents sont aussi des « technocrates » au sens que donnait à ce terme Alvin Gouldner (1979), c'est-à-dire des membres de catégories sociales mettant l'accent, dans leur activité professionnelle, sur les liens entre connaissance, action et pouvoir. L'encerclement qu'ils privilégient comprend un ensemble de pratiques visant à restreindre l'univers des choix des enfants, afin que ces derniers ne dévient pas fortement par rapport aux projets parentaux.

\section{Des stratégies de contrôle de l'environnement}

Cette orientation correspond à des visées utilitaristes en matière de scolarisation, mettant l'accent sur la valeur d'échange du capital scolaire sur le marché de l'enseignement supérieur et du travail mais aussi sur d'autres marchés sociaux, comme le marché matrimonial (Collins, 1979). Ces visées utilitaristes se doublent néanmoins de visées expressives mettant l'accent sur l'importance de l'acquisition de " savoir être » liés à l'exercice du pouvoir dans l'activité professionnelle, comme le leadership ou l'esprit de compétition, et à la reproduction des positions de classe, comme les bonnes manières. Cette logique renvoie également à des valeurs orientées vers l'égalité formelle des chances scolaires et sociales et vers une intégration sociale " par cloisonnement ", c'est-à-dire par l'insertion des individus dans des groupes sociaux eux-mêmes bien insérés dans un ordre hiérarchisé.

Le recours à l'encerclement s'accompagne en outre d'une importance particulière accordée à l'environnement social dans la construction de la personnalité. L'inculcation des connaissances et des « savoir être » est perçue comme étroitement dépendante de l'entourage de l'enfant, et ce dernier comme très sensible aux interactions sociales dans ses divers univers de socialisation. De ce fait, les pratiques parentales varient fortement suivant que l'enfant évolue dans un environnement homogène, avec des enfants semblables à lui, ou qu'il se trouve confronté à un environnement hétérogène, perçu comme défavorable à une bonne socialisation. Dans le premier cas, qui caractérise les familles les plus proches du modèle de la grande bourgeoisie, l'encerclement relève de " l'allant de soi » (Pinçon et PinçonCharlot, 2007). Dans le second, plus fréquent, il se traduit par des pratiques conscientes visant à créer et à maintenir des barrières de protection. 
Plus les parents s'inscrivant dans la logique de l'encerclement sont anxieux vis-à-vis de l'entourage de l'enfant, plus ces choix sont précoces et radicaux et impliquent une très forte mobilisation « protectrice » autour de l'enfant. Cette dernière commence, dans certaines familles, avant ou peu de temps après la naissance des enfants, par des stratégies résidentielles visant à recréer un entre soi de classe. Elle se poursuit au travers des choix concernant la garde de l'enfant, si la mère travaille, ou même si elle ne travaille pas mais qu'elle estime utile pour elle et pour son enfant de le confier quelques heures à une autre personne. Les ressources économiques ainsi que l'attention portée à l'entourage conduisent très souvent, dans ce cas, à délaisser les crèches publiques au profit de nounous, si possible hébergées ou accueillies à la maison (Ball et Vincent, 2006). Ce contrôle de l'environnement s'objective, par la suite, dans une myriade de décisions plus ou moins visibles, qui vont du choix des camarades de l'enfant qui sont invités à la maison, à des sorties ou aux anniversaires, au choix des activités extrascolaires, culturelles ou sportives. Tous ces choix sont orientés par des visées instrumentales et expressives mais aussi sociales, c'est-à-dire par le souci de contrôler les caractéristiques des autres enfants et donc, dans l'esprit de ces parents, le contenu des interactions.

\section{Des enjeux autour de l'enseignement scolaire}

En raison des enjeux dont elle est porteuse, c'est toutefois autour de la scolarisation que ces stratégies de contrôle de l'environnement se déploient de la façon la plus active. Si les choix résidentiels de ces familles sont souvent liés à la volonté de se rapprocher des meilleurs établissements publics soumis à des procédures de sectorisation, ces derniers sont parfois fuis à la faveur d'établissements privés, plus sélectifs socialement et proposant un traitement " sur mesure », deux conditions perçues comme favorables à l'éclosion des « savoir être » souhaités chez l'enfant (Ballion, 1980). Ces choix d'établissements se doublent de choix de filières et d'options orientés par la même recherche d'entre soi, en même temps que de signes distinctifs de «qualité » pouvant servir de repères pour les futurs enseignants et les futurs employeurs (Collins, 1979). S'y ajoutent des stratégies fondées sur le recours à des prestations commerciales, comme les cours particuliers - voire, dans certains cas, le coaching - et les séjours à l'étranger, ayant pour but d'anticiper les difficultés ainsi que de préparer à l'insertion dans des contextes d'étude et de travail compétitifs et, aussi, de plus en plus internationalisés.

Comment s'y prennent ces familles des classes supérieures pour faire accepter ces choix à leurs enfants sans déroger au respect pour l'autonomie enfantine et adolescente qu'elles affichent? L'analyse de leurs échanges avec leurs enfants montre qu'elles privilégient deux vecteurs : le contrôle et la moralisation (Kellerhals et Montandon, 1991). La place accordée au contrôle les rapproche à la fois des catégories populaires et des indépendants (voir l'arti- 
cle de Marie-Clémence Le Pape dans ce numéro, page 88). Elles s'en distinguent cependant par l'invisibilité relative de leurs pratiques. Les choix ne sont pas imposés aux enfants car le contrôle est souvent délégué à l'entourage, c'est-à-dire à la fois à d'autres adultes que l'on a choisis, mais aussi au cadre lui-même et notamment aux effets de l'entre soi enfantin ou adolescent. Par ailleurs, au contrôle se superpose la moralisation, qui vise à rendre l'enfant responsable face à des choix qui mettent souvent en balance le bonheur et la réussite, le plaisir présent et l'intérêt à long terme. Ce mode de contrôle se révèle efficace si les parents, et notamment les mères, sont en mesure d'assumer le coût matériel mais également symbolique que représente l'érection permanente de barrières et de mettre progressivement les enfants de leur côté. Car, aussi subtil que soit le cadrage, il ne passe pas inaperçu des enfants qui, en grandissant, s'ils n'adhèrent pas aux normes et aux attentes du milieu dans lequel ils sont insérés, peuvent se révolter de façon plus ou moins violente et adopter des choix déviants par rapport aux attentes parentales.

\section{L'argumentation ou l'effort pour convaincre les enfants}

Les « intellectuels », c'est-à-dire les spécialistes de l'élaboration et de la transmission des idées, ont plutôt recours à une autre stratégie : l'argumentation (Gouldner, 1979). Les membres de cette catégorie, qui se recrutent principalement parmi les chercheurs, les journalistes, les enseignants ou les éducateurs, mettent en œuvre des pratiques dont la cohérence découle d'une certaine conception de la rationalité. Cette dernière n'est pas exclusivement de nature utilitariste. Si ces parents insistent auprès de leurs enfants sur la nécessité, en termes d'insertion professionnelle, de détenir un capital culturel objectivé par des notes, des classements et des diplômes, la connaissance, entendue ici comme un rapport lucide aux autres et aux choses, est valorisée en soi comme une composante indispensable du développement personnel et de celui de l'humanité tout entière.

\section{Des choix éducatifs pour l'épanouissement de l'enfant}

Ces visées réflexives sont par ailleurs associées à des visées expressives qui mettent moins l'accent sur l'éclosion de la personnalité que sur le bonheur et notamment celui lié à la relation avec les autres. On peut néanmoins, à cet égard, établir une ligne de division entre les classes intellectuelles supérieures et moyennes. Chez les premières, un subtil dosage d'utilitarisme et de réflexivité, qui met le bonheur immédiat au deuxième rang, oriente les pratiques. Chez les secondes, l'« épanouissement » de l'enfant est souvent privilégié, en donnant la priorité, au moins jusqu’à la fin des années collège, au « moratoire expressif ». De façon plus modérée dans le premier groupe, plus importante dans le second, ces visées sont généralement associées à un idéal d'égalité impliquant la mise en place d'actions destinées à compenser les injustices 
associées aux positions sociales d'origine et à une vision de l'intégration reposant sur le côtoiement d'autres différents de soi. Ces conceptions ne conduisent cependant pas à la promotion d'une société égalitaire et ouverte à toutes les cultures, dans la mesure où la valorisation de la connaissance va souvent de pair avec des hiérarchies et des séparations fondées sur les certifications scolaires et sur la maitrise de certaines formes culturelles.

La préservation des conditions favorables au développement intellectuel et personnel est chez ces parents une priorité, mais, contrairement au groupe précédent, elle n'induit pas automatiquement la reconstitution d'un entre soi. En effet, ces parents ont confiance dans la compétence professionnelle des spécialistes de la connaissance et de l'humain (puéricultrices, moniteurs, enseignants...), à qui ils délèguent partiellement l'éducation de leurs enfants. Cette confiance repose sur la forte similitude des habitus sociaux et des idéologies professionnelles et politiques ainsi que sur la mise en œuvre de formes de coopération efficaces avec ces professionnels (Dubet et Martucelli, 1996). À cela s'ajoute la confiance qu'ont ces parents dans la capacité d'autorégulation de leurs enfants. Cette capacité est continuellement entretenue par des échanges avec eux, visant à les doter des compétences intellectuelles et sociales nécessaires à résister aux dangers et aux possibles influences négatives de leur environnement.

Les choix éducatifs auxquels procèdent ces parents sont, davantage que chez les parents « managers », guidés par l'intérêt intellectuel qu'ils attribuent aux différents contextes de socialisation ainsi que par la qualification et la compétence des professionnels qui en ont la charge, qu'il s'agisse des crèches collectives publiques qu'ils sont plus nombreux à utiliser que les parents du groupe précédent, ou des activités extrascolaires des enfants. Dans ce dernier cas, prime l'acquisition de savoirs et de savoir-faire favorisant, dans leur esprit, le développement intellectuel et physique des enfants et la reproduction d'un certain modèle de civilisation, mais aussi de compétences sociales (échanger et travailler avec d'autres) et politiques (militer en faveur de certaines causes). Les goûts de l'enfant sont davantage pris en compte que chez les parents du groupe précédent, mais ils font l'objet d'un modelage en douceur par des stratégies de requalification et de prise de distance réflexive. Les parents peuvent ainsi acheter des bandes dessinées ou des jeux vidéo mais en choisissant ceux qui leur semblent le plus à même de développer diverses capacités intellectuelles. Ils sont susceptibles, dans le même esprit, de regarder avec leurs enfants un programme de télévision qu'ils jugent peu intéressant, mais en faisant appel, pendant ou après, à la capacité de jugement de ces derniers. Le projet, à long terme, est l'abandon progressif par les enfants de certaines pratiques, grâce à l'exercice de leurs compétences critiques ou la constitution d'une culture personnelle faite d'un bricolage intellectuel d'usages relevant de la culture légitime et du simple divertissement (Lahire, 2004). 


\section{L'accompagnement scolaire}

Sur le plan scolaire, les écoles primaires et les collèges du secteur sont souvent privilégiés en raison d'un attachement au quartier qui mêle des dimensions expressives - le bien-être associé à la proximité et le bonheur associé à la sociabilité locale - et axiologiques - se comporter en citoyen responsable en favorisant ainsi l'intégration à l'école et par l'école (Raveaud et van Zanten, 2007). Toutefois, du fait que ces parents habitent souvent des quartiers " mélangés ", certaines conditions doivent être réunies, dont la principale est la présence d'une proportion jugée suffisante d'enfants du même milieu social que les leurs. À cela s'ajoute, chez certains, un investissement destiné à compenser les manques de l'établissement local par l'obtention de ressources financières ou humaines supplémentaires et diverses actions en direction des enfants le plus en difficulté (van Zanten, 2007). Chez d'autres, il s'agit plutôt de suppléer à ces carences par la reconstitution d'un entre soi académique et social dans des classes de niveau. Cet entre soi, plus poreux que celui obtenu par le choix résidentiel ou celui du privé, ne suffit cependant pas aux plus stratèges d'entre eux, qui optent alors pour des établissements publics hors secteur (van Zanten, 2001). Dans tous les cas, on observe un accompagnement tout aussi intense sinon plus de la scolarité de l'enfant que chez les catégories précédentes. Toutefois, cet accompagnement passe moins par le recours à des instances extérieures que par une organisation de la vie familiale dans le but de permettre aux parents, et surtout aux mères, de se rendre disponibles pour soutenir les efforts scolaires des enfants.

Cette disponibilité est essentielle pour ces familles que B. Bernstein (1975) qualifie de « relationnelles » car les échanges sont leur outil privilégié pour canaliser les choix des enfants. Ces parents agissent en effet au travers de subtiles négociations dans lesquelles le point de vue des enfants ou des adolescents est pris en compte mais intégré dans un débat caractérisé par l'asymétrie des positions et des ressources. Ils n'excluent pas d'emblée les choix scolaires et professionnels des enfants, mais quand ceux-ci semblent dévier de ce qu'ils souhaitent pour eux, ils cherchent à les infléchir en énonçant les compatibilités et les incompatibilités présentes et futures par rapport à des visées de réalisation de soi et d'estime sociale, en précisant les efforts à fournir et les gains à espérer, en pointant les limites ou les effets pervers. Bref, ils cherchent à convaincre leurs enfants en leur apprenant, par touches successives, à modifier la balance des coûts et profits subjectifs (Kellerhals et Montandon, 1991 ; Le Pape et van Zanten, 2009). Certains d'entre eux sont néanmoins pris au piège de ces méthodes de socialisation « délibératives » dès lors que leurs enfants, devenus experts en la matière, leur présentent des choix qu'ils se révèlent incapables de contrecarrer sans recourir à l'imposition, signant ainsi un échec de leur mode indirect d'encadrement. 
L'analyse du travail parental chez les classes moyennes et supérieures montre la tension que suscite la coexistence de deux visées difficilement conciliables, à savoir, d'une part, le respect d'une autonomie enfantine et adolescente à laquelle ces catégories sociales se déclarent fortement attachées (Singly, 2000) et, d'autre part, la nécessité d'encadrer des pratiques et des choix qu'ils estiment cruciaux pour l'avenir de leurs enfants et la reproduction de leur propre position sociale. Les ressources économiques, culturelles et sociales dont disposent ces familles leur permettent néanmoins, dans de nombreux cas, de modeler ces choix de façon discrète et continue, en suscitant peu de contestations directes de la part des adolescents. Ce « modelage » prend néanmoins des formes différentes selon qu'il s'agit de parents proches du pôle des " managers » ou de celui des « intellectuels » et que les méthodes mobilisées relèvent davantage de la structuration de l'environnement ou de l'appel à la raison. Or, bien qu'il ne soit pas possible d'en apporter la preuve dans cet article, il apparaît fondé de supposer que ces modes de contrôle ont des effets distinctifs durables non seulement dans le domaine de l'éducation familiale et scolaire mais dans bien d'autres, et qu'ils contribuent ainsi au maintien de clivages importants au sein des élites au pouvoir, des groupes professionnels ou des communautés locales.

\section{Bibliographie}

- Ball S. et Vincent C., 2006, Childcare, Choice and Class Practices : MiddleClass Parents and Their Children, Londres, Routledge.

- Ballion R., 1980, "L'enseignement privé : une école "sur mesure" ? ", Revue française de sociologie, vol. 21, p. 203-231.

- Bernstein B., 1975, Langages et classes sociales. Codes socio-linguistiques et contrôle social, Paris, Éditions de Minuit ; 2007, Pédagogie, contrôle symbolique et identité. Théorie, recherche, critique, Laval, Presses universitaires de Laval.

- Collins R., 1979, The Credential Society. A Historical Sociology of Education, New York, Academic Press.

- Dubet F. et Martucelli D., 1996, " Les parents et l'école : classes populaires et classes moyennes ", Lien social et politique, $\mathrm{n}^{\circ}$ 35, p. 109-121.

- Gombert P., 2008, L'école et ses stratèges. Les pratiques éducatives des nouvelles classes supérieures, Rennes, Presses universitaires de Rennes.

- Gouldner A., 1979, The Future of Intellectuals and the Rise of the New Class, New York, The Seabury Press.

- Hays S., 1996, The Cultural Contradictions of Motherhood, New Haven et Londres, Yale University Press. 
- Kellerhals J. et Montandon C., 1991, Les stratégies éducatives des familles. Milieu social, dynamique familiale et éducation des pré-adolescents, Lausanne, Delachaux et Niestlé.

- Lahire B., 2004, La culture des individus : dissonances culturelles et distinction de soi, Paris, La Découverte.

- Lareau A., 2003, Unequal Childhoods. Class, Race and Family Life, Berkeley, University of California Press.

- Le Pape M.-C. et van Zanten A., 2009, « Les pratiques éducatives des familles ", in Duru-Bellat M. et van Zanten A., Sociologie du système scolaire. Les inégalités d'éducation, Paris, Presses universitaires de France.

- Pinçon M. et Pinçon-Charlot M., 2007, Sociologie de la bourgeoisie, Paris, La Découverte.

- Raveaud M. et van Zanten A., 2007, "Choosing the Local School ? Middle Class Parents' Values and Social and Ethnic Mix in London and Paris », Journal of Education Policy, vol. 22, n 1, p. 107-124.

- Singly F. de, 2000, Libres ensemble : I'individualisme dans la vie commune, Paris, Nathan.

- van Zanten A., 2001, L'école de la périphérie : scolarité et ségrégation en banlieue, Paris, Presses universitaires de France ; 2007, " Individualisme et solidarité dans les choix éducatifs des familles ", in Paugam S. (dir.), Repenser la solidarité. L'apport des sciences sociales, Paris, Presses universitaires de France ; 2009 (à paraître), Choisir son école. Stratégies individuelles et médiations locales, Paris, Presses universitaires de France. 